\title{
Progression to metastatic disease from a small renal cell carcinoma prospectively followed with an active surveillance protocol
}

\author{
Jaime A. Wong, MD; Ricardo A. Rendon, MD
}

\begin{abstract}
With the widespread use of abdominal imaging to evaluate other medical conditions, many renal tumours are being diagnosed at earlier stages. Older patients have experienced the most significant increase in the incidence of renal cell carcinoma (RCC). This age group frequently has significant medical comorbidities. This has led to the concept of active surveillance for select patients with renal lesions that may not affect their mortality. However, the ultimate risk of active surveillance is the potential for developing metastases. This case report presents the development of metastatic disease from a small, incidentally detected and prospectively followed RCC with asymptomatic progression.
\end{abstract}

CUAJ 2007;1(2):120-2

$\mathrm{T}$ he incidence of renal cell carcinoma (RCC) is rising. It accounts for $3 \%$ of all adult malignancies. ${ }^{1}$ Patients aged $70-90$ years have experienced the most significant increase., ${ }^{2,3}$ This age group frequently has significant medical comorbidities. With the more common use of abdominal imaging to evaluate other medical conditions, many renal tumours are being diagnosed at earlier stages. ${ }^{2}$ The presentation of RCC by incidental detection has increased from $10 \%$ to greater than $60 \%$ over the past 30 years. ${ }^{4}$ This has led to the concept of active surveillance for select patients with renal lesions that may not affect their mortality. The ultimate risk of active surveillance is, however, the potential for developing metastasis, at which point curative treatment is no longer an option. This case report presents a patient with a small RCC who developed metastatic disease while on active surveillance.

\section{Case Presentation and Management}

A 78-year-old woman presented with a $3 \times 3$-cm upper lobe lung lesion diagnosed on a chest $\mathrm{x}$-ray. Her past medical history was significant for aortic stenosis, hypertension, chronic obstructive pulmonary disease, type II diabetes mellitus and dyslipidemia. A CT scan revealed a $2 \times$ $2.5-\mathrm{cm}$ lesion in the upper pole of her left kidney. This represented an estimated tumour volume (ETV) of $6.0 \mathrm{~mL}$ as calculated by the formula $0.5326 x y(x+y / 2) .{ }^{5}$ There were no other signs of metastases. The patient was asymptomatic with respect to her renal mass and had no hematuria. Both of the lesions were biopsied. The lung lesion was a nonsmall cell lung cancer, and the renal lesion was a clear cell RCC (Fuhrman grade 1-2/4).

The patient was offered surgery or radiotherapy for the lung lesion.
She chose radiotherapy because she wished to avoid surgery. Given the small size of the renal lesion, the patient's competing health risks and her desire to avoid surgery, she chose active surveillance for the RCC.

After radiotherapy, the lung lesion decreased in size significantly and it was felt that the patient had a good response. A CT scan performed at 3-month follow-up revealed an increase in size of the renal mass to $3.5 \times 3 \mathrm{~cm}$ $(E T V=18.2 \mathrm{~mL})$. Further imaging was performed every 3 months. The renal lesion remained stable in size until the 15 -month follow-up, when it showed significant growth. The mass measured $5.9 \times 5.4-\mathrm{cm}(\mathrm{ETV}=95.9$ $\mathrm{mL}$ ) and was associated with extensive retroperitoneal lymphadenopathy (Fig. 1). In addition, new nodular densities consistent with metastatic disease were noted in both lungs. In this $\mathrm{CT}$, the original lung lesion measured $<1 \mathrm{~cm}$. The patient had been asymptomatic with respect to her renal lesion at the time of this progression. The patient declined biopsy of the metastatic deposits. Given the rapid increase in the size of the renal lesion, the associated retroperitoneal lymphadenopathy and the good response to radiation of the original lung lesion, the metastatic lesions were presumed to be of RCC origin. The patient declined immunotherapy. She died 5 months later, and an autopsy was refused by her family.

\section{Discussion}

Most of the data regarding active surveillance for RCCs have been reported over the past decade. Overall, there have been 10 small series regarding expectant management for renal masses that have included 286 lesions. ${ }^{6}$ A recent meta-analysis of enhancing renal masses managed expectantly was performed on 234 of these lesions, which demonstrated 
an overall mean initial lesion size of $2.60 \mathrm{~cm}$ and a mean growth rate of $0.28 \mathrm{~cm}$ annually, with a mean follow-up of 34 months. ${ }^{6}$ The growth rates of the lesions were not dependant upon the size of the lesions at presentation. ${ }^{6}$ Most of the lesions that were removed were malignant, but this may represent a selection bias as rapidly growing lesions are more likely to be removed, compared with those that are stable in size or slow-growing.

Absolute indications for active surveillance include patient refusal of surgery and patients for whom surgery is contraindicated owing to severe medical comorbidities making surgical risk unacceptable. Relative indications include concomitant disease, such as a second malignancy or significant medically manageable illness, or patients for whom surgical extirpation may result in the necessity of renal replacement therapy. ${ }^{1}$

Metastases secondary to small renal lesions are unusual. In Bosniak's series of 100 patients with proven $\mathrm{RCC}<3 \mathrm{~cm}$, only 2 patients demonstrated metastases.' Both of these patients presented with symptoms associated with metastases and their renal lesions were found upon searching for the primary malignancy. Bosniak suggested that most tumours do not metastasize until they reach $3-4 \mathrm{~cm}$ in size. ${ }^{7}$ Curry and colleagues reported the highest rate of metastases in patients with renal masses $<3 \mathrm{~cm}^{8}{ }^{8}$ However, this series included larger masses and consisted of only 9 patients, with 2 of these patients (22\%) developing metastases at 2 and 4 years after diagnosis. The first patient presented with metastases and a $1.5 \mathrm{~cm}$ renal lesion was discovered upon searching for the primary lesion. The second patient was originally diagnosed with a benign renal cyst, despite the lesion having elevated Hounsfield numbers, heterogeneity and an indistinct parenchymal interface. Unfortunately, the patient did not have follow-up. The patient presented 4 years later with metastases to the adrenal gland and regional lymphadenopathy. Both of these patients had hematuria. ${ }^{8}$ Other studies of small renal masses have documented concurrent metastases in $5 \%-7 \%$ of patients. ${ }^{9}$

Progression to metastatic disease has only been reported in 3 patients with RCC on expectant management. The first patient developed metastases at 132 months follow-up. ${ }^{10}$ The initial size of the lesion was not reported. The patient had a biopsy

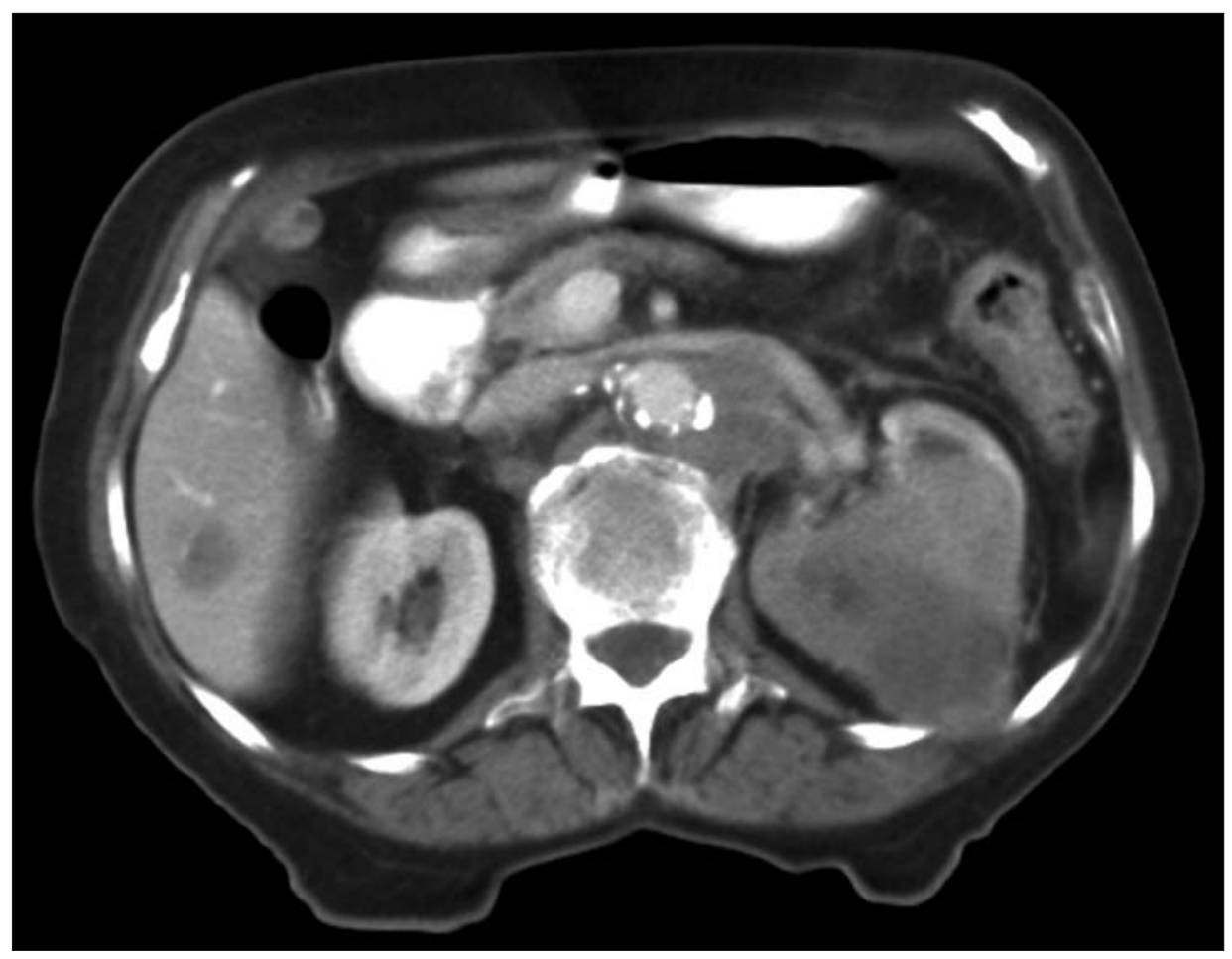

Fig. 1. CT scan of the abdomen demonstrating a left renal mass associated with extensive retroperitoneal lymphadenopathy. 
Wong and Rendon

negative for malignancy and was lost to follow-up. The patient subsequently presented with hematuria and evidence of metastatic disease. ${ }^{10}$ Sowery and Siemens reported the case of the second patient, who developed metastatic disease at 111 months. ${ }^{4}$ The initial lesion was $8.8 \mathrm{~cm}$ and had a growth rate of $0.2 \mathrm{~cm}$ annually. This patient required embolization treatments on 2 occasions for recurrent hematuria. The final patient, described by Chawla and colleagues, was 84 years old and presented with a $2.0 \mathrm{~cm}$ lesion. ${ }^{6}$ The lesion grew rapidly at a rate of $1.3 \mathrm{~cm}$ annually until it reached a final diameter of $8 \mathrm{~cm}$. Metastatic disease developed at 54 months of follow-up. All 3 patients were symptomatic at the time of their disease progression. ${ }^{4,6,10}$

To the best of our knowledge, this is the first report of the development of metastatic disease from a small, incidentally detected and prospectively followed RCC with asymptomatic progression. This patient was followed with serial imaging every 3 months. This highlights the need for continued serial radiological imaging, even in asymptomatic patients. Most small incidental tumours are associated with a slow natural growth rate and low metastatic risk. ${ }^{6}$ However, metastases represent the most significant risk of surveillance in patients with RCC because the treatment options for metastatic RCC have limited success. With the growing enthusiasm and implementation of active surveillance for RCC, we wish to report this case to confirm that, although the risk of metastases from small renal masses is low, it is not negligible. We must ensure that active surveillance is only employed in appropriate patients with compet- ing health risks and we must be diligent with our follow-up because metastases remain a rare but potential outcome.

From the Department of Urology, Dalhousie University, and the Queen Elizabeth II Health Sciences Centre, Halifax, NS.

This article has been peer reviewed.

Competing interests: None declared.

\section{References}

1. Kassouf $W$, Aprikian AG, Laplante $M$, et al. Natural history of renal masses followed expectantly. J Urol 2004;171:111-3.

2. Chow WH, Devessa SS, Warren JL, et al. Rising incidence of renal call cancer in the United States. JAMA 1999;281:1628-31.

3. Katz DL, Zheng T, Holford TR, et al. Time trends in the incidence of renal cell carcinoma: analysis of Connecticut tumor registry data, 1935-1989. Int I Cancer 1994;58:57-63.

4. Sowery RD, Siemens DR. Growth characteristics of renal cortical tumors in patients managed by watchful waiting. Can I Urol 2004;11:2407-10.

5. Volpe A, Panzarella T, Rendon RA, et al. The natural history of incidentally detected small renal masses. Cancer 2004;100:738-45.

6. Chawla SN, Crispen PL, Hanlon AL, et al. The natural history of observed enhancing renal masses: meta-analysis and review of the world literature. J Urol 2006;175:425-31.

7. Bosniak MA. Observation of small incidentally detected renal masses. Semin Urol Oncol 1995; 13:267-72.

8. Curry NS, Schabel SI, Betsill WL Jr. Small renal neoplasms: diagnostic imaging, pathologic features and clinical course. Radiology 1986;158:113-7.

9. Curry NS, Biassada NK. Radiologic evaluation of small and indeterminant renal masses. Urol Clin North Am 1997;24:493-505.

10. Lamb GW, Bromwich EJ, Vasey P, et al. Management of renal masses in patients medically unstable for nephrectomy-natural history, complications, and outcomes. Urology 2004;64:909-13.

Correspondence: Dr. Ricardo A. Rendon, Queen Elizabeth II Health Sciences Centre, Rm. 210, 5-South, Victoria Building, 1278 Tower Road, Halifax NS B3H 2Y9; fax 902 492-2437; rrendon@dal.ca 